\title{
Design of constant gain UWB planar antenna using FSS-based reflectors
}

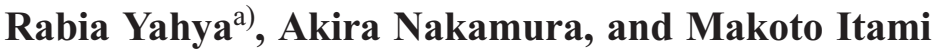 \\ Department of Applied Electronics, Tokyo University of Science, \\ 6-3-1 Niijuku, Katsushika-ku, Tokyo 125-8585, Japan \\ a)rabia@itamilab.te.noda.tus.ac.jp
}

\begin{abstract}
In this letter, a technique to improve the gain of ultra-wideband (UWB) planar antennas, by using low-profile frequency selective surface (FSS) based reflectors, is proposed. This technique not only enhances the gain of the planar UWB antennas but also guarantees a constant gain of maximum variation of $0.5 \mathrm{~dB}$ across the entire UWB, while keeping their attractive merits. To prove the effectiveness of the proposed technique, UWB coplanar waveguide (CPW) fed circular-disc antenna is installed above the proposed reflectors. As result, constant gain UWB antenna has been obtained.
\end{abstract}

Keywords: constant gain antennas, UWB directional antennas, FSS reflectors

Classification: Antennas and Propagation

\section{References}

[1] J. Liang, C. C. Chiau, X. Chen, and C. G. Parini, "Printed circular disc monopole antenna for ultra-wideband applications," Electron. Lett., vol. 40, no. 20, pp. 1246-1247, September 2004. DOI:10.1049/el:20045966

[2] Y. F. Liu, K. L. Lau, Q. Xue, and C. H. Chan, "Experimental studies of printed wide-slot antenna for wide-band applications," IEEE Antennas Wireless Propag. Lett., vol. 3, no. 1, pp. 273-275, 2004. DOI:10.1109/LAWP.2004. 837510

[3] J. Liang, L. Guo, C. C. Chiau, X. Chen, and C. G. Parini, "Study of CPW-fed circular disc monopole antenna for ultra wideband applications," IEE Proc. Microwaves Antennas Propag., vol. 152, no. 6, pp. 520-526, December 2005. DOI:10.1049/ip-map:20045179

[4] S. S. Zhong, X. L. Liang, and W. Wang, "Compact elliptical monopole antenna with impedance bandwidth in excess of 21:1," IEEE Trans. Antennas Propag., vol. 55, no. 11, pp. 3082-3085, November 2007. DOI:10.1109/TAP.2007. 908565

[5] X. Qing and Z. N. Chen, "Compact coplanar waveguide-fed ultra-wideband monopole-like slot antenna," IET Microwaves Antennas Propag., vol. 3, no. 5, pp. 889-898, 2009. DOI:10.1049/iet-map.2008.0075

[6] S. Wang, X. D. Chen, and C. G. Parini, "Analysis of ultra wideband antipodal Vivaldi antenna design," Proc. 2007 Conf. Antennas and Propagation, Loughborough, England, pp. 129-132, April 2007. DOI:10.1109/LAPC. 2007.367448 
[7] A. Locatelli, D. Modotto, F. M. Pigozzo, S. Boscolo, E. Autizi, C. De Angelis, A. D. Capobianco, and M. Midrio, "Highly directional planar ultra wide band antenna for radar applications," Proc. $37^{\text {th }}$ Conf. European Microwave Integrated Circuit Conference, Munich, Germany, pp. 1421-1424, October 2007. DOI:10.1109/EUMC.2007.4405471

[8] M. Mokhtaari and J. Bornemann, "Directional ultra-wideband antennas in planar technologies," Proc. $38^{\text {th }}$ Conf. European Microwave Conference, Amsterdam, Netherlands, pp. 885-888, October 2008. DOI:10.1109/EUMC. 2008.4751595

[9] Y. Ranga, L. Matekovits, A. R. Weily, and K. P. Esselle, "A Constant gain ultra-wide band antenna with a mlti-layer frequency selective surface," PIER Letters, vol. 38, pp. 119-125, 2013. DOI:10.2528/PIERL13021007

[10] Y. Ranga, L. Matekovits, K. P. Esselle, and A. R. Weily, "Multioctave frequency selective surface reflector for ultra wideband antennas," IEEE Antennas Wireless Propag. Lett., vol. 10, pp. 219-222, 2011. DOI:10.1109/ LAWP.2011.2130509

[11] First Report and Order, Revision of Part 15 of the Commission's Rules Regarding Ultra-Wideband Transmission Systems, Federal Communications Commission, ET Docket 98-153, FCC 02-48, Feb. 2002.

[12] M. Pasian, S. Monni, A. Neto, M. Ettorre, and G. Gerini, "Frequency selective surfaces for extended bandwidth backing reflector functions," IEEE Trans. Antennas Propag., vol. 58, no. 1, pp. 43-50, January 2010. DOI:10.1109/TAP. 2009.2036185

[13] J. C. Vardaxoglou, R. Lee, and A. Chauraya, "Frequency-selective surface and electromagnetic bandgap theory basics" in Theory and Phenomena of Metamaterials, ed. F. Capolino, pp. 30-1-30-27, CRC Press, Taylor and Francis Group, Boca Raton, 2009. DOI:10.1201/9781420054262

[14] Y. Rahmat-Samii and F. Yang, "Development of complex artificial ground planes in antenna engineering" in Metamaterials Physics and Engineering Explorations, ed. N. Engheta and R. W. Ziolkowski, pp. 315-349, John Wiley \& Sons, Inc, 2006. DOI:10.1002/0471784192

\section{Introduction}

Over the last decades, UWB technology has gained a lot of attention as consequence, of its ability to optimize the performance of the existing systems and open the doors widely for future applications and as results, to the attractive merits that it provides such as wide bandwidth, high data rate, interference mitigation and low transmission power. To benefit in the best ways of this technology, UWB antennas providing valuable characteristics such as planar structures, compact size and easy fabrication using printed circuit board (PCB) technology, have been proposed [1, 2, $3,4,5]$. However, the provided radiation is omnidirectional and bidirectional in the principal planes. Although, this is desirable for the applications where the users' locations are not predefined as communication systems, a directional radiation is more suitable for other applications such as radar and medical applications. Accordingly, the radiation nature of the omnidirectional planar UWB antennas restricts them to be used for the applications that require directional radiation. Subsequently, immense research has been devoted to offer UWB planar antennas with directional radiation whether, by developing conventional directional broad- 
band antennas [6] or by proposing some approaches and techniques contribute to provide antennas with these desirable features $[7,8,9,10]$. [7, 8] provide axial directional radiation and $[9,10]$ provide broadside directional radiation using multilayer FSS reflectors. Comparing with these reflectors, the proposed reflectors inhere are FSS structures construct with sub-wavelength cells printed on single dielectric substrate with and without perfect electric conductor (PEC) ground, which simplifies the implementation and minimizes the profile of the overall structure. In this paper, firstly, we propose grounded FSS reflector to serve the purpose of high-gain planar structure as well as constant gain across the entire band from 3.1 to $10.6 \mathrm{GHz}$ which is the band authorized by the FCC for UWB applications in 2002 [11]. Thereafter, a further improvement is made to derive a single-layer FSS occupies one side of substrate which eliminates the need for ground plane. The effectiveness of the proposed reflectors to improve the gain of the UWB omnidirectional planar antennas is proved by means of using UWB antenna as radiator. The design and optimization process has been performed using computer simulation technology (CST-MWS).

\section{Design process}

The main objective of this letter is to design reflectors to be able to reflect the incident waves over the entire UWB band. PEC ground plane can be used as reflector, but its performance cannot be guaranteed over broadband frequency range as UWB band especially over the high frequencies. To alleviate this limitation, FSS can be employed [12]. As result, grounded FSS will serve as UWB reflector. In addition, this reflector needs to have a reflection coefficient varies with frequency in a manner stabilizes the gain of the UWB antennas over the UWB band. Therefore, we use grounded FSS with high resonance frequency to obtain the both characteristics.

\subsection{Grounded FSS reflector}

Frequency selective surfaces are 2D periodic arrays of metallic elements or apertures that exhibit stop-band or pass-band characteristics when excited by an electromagnetic wave at an angle arbitrary to the plane of the array. FSS with array of conductors is fully reflective at resonance frequency where it acts as a metallic sheet and it remains transparent at other frequencies [13]. This feature can be used to design partially reflector with desirable reflection coefficient by choosing the location of its resonance frequency. High resonance frequency FSS with array of conductors can act as transmitter with reflection magnitude increases with frequency. Thereafter, the transmitted waves can be reflected totally using PEC ground plane. This way the grounded FSS will improve the gain of used radiator across the entire UWB band with different amount of enhancement, hence; a constant gain can be achieved. Grounded FSS can also be considered as grounded dielectric slab loaded with periodic patches, which is similar to high impedance surface (HIS) with removed vertical vias. Therefore, we used the design process suggested in [14] and CST-MWS with considering the "unit cell" boundary conditions and Floquet ports, to design grounded slab loaded with periodic square patches of width $\mathrm{W}$ and 
gap width $g$ between the unit cells as indicated in Fig. 1.a, to have a widest in-phase band centered at $7.5 \mathrm{GHz}$. Furthermore, a foam substrate of dielectric constant of 1.07 and thickness of $\mathrm{hu}=3 \mathrm{~mm}$ has been chosen. It has been revealed that the reflection coefficient magnitude of this unit cell without the ground plane increases with the frequency and it is totally reflective around $45 \mathrm{GHz}$. Over the UWB band, the magnitude of the reflection coefficient varies from 0.3 at lower frequency to 0.8 at higher frequency. As result, the reflected amount by the ground plane is unequal and is proportionally decreased with frequency. This is the key feature to achieve constant gain across the entire UWB, especially if the gain of the used radiator is higher at upper frequencies than it is at lower frequencies which is the case of the most part of UWB planar antennas. In [9], this feature is achieved by allowing the transmission through no backed multilayer FSS, inhere; it is achieved with only one partially reflective FSS.

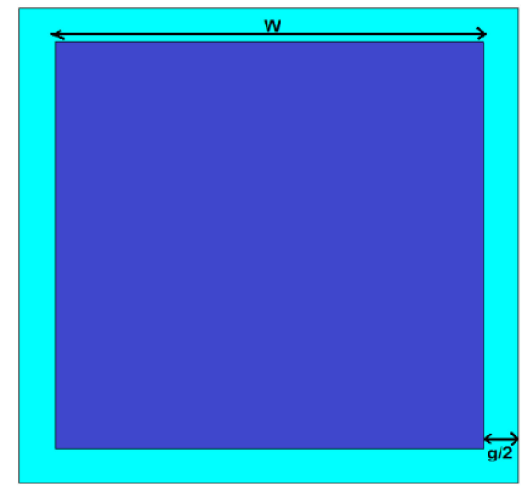

(a) FSS unit cell

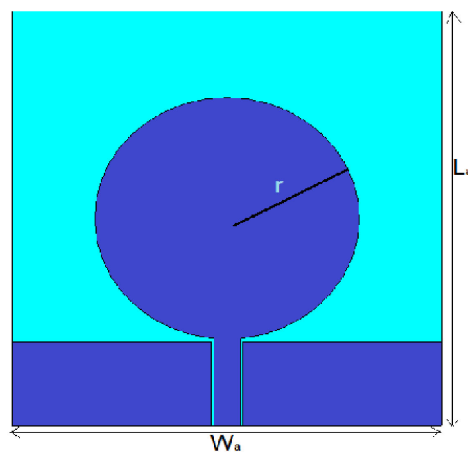

(c) UWB antenna above reflector

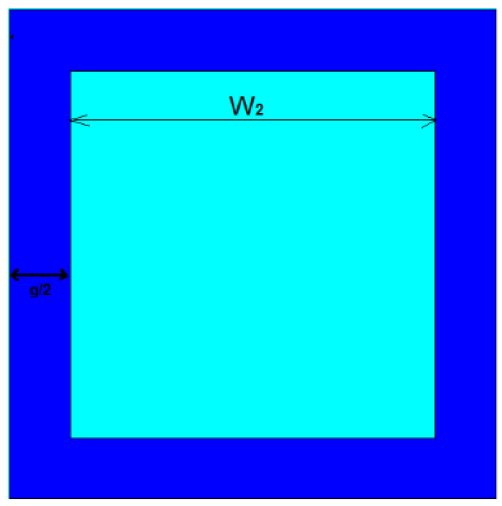

(b) Complementary unit cell

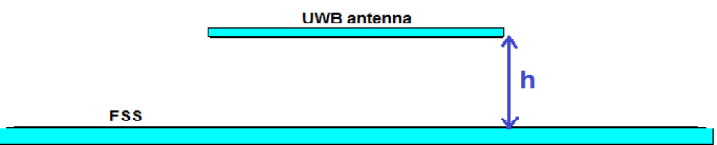

Fig. 1. Structures of the proposed FSS unit cells and the used UWB antenna.

\subsection{Single-layer FSS reflector}

The operation mechanism of the grounded FSS reflector treated in the last section can be realized differently in manner leads to further improvement of the proposed approach, by using complementary structure. The complementary structure of the previous used FSS is a FSS with array of square slots printed on the same substrate 
as shown in Fig. 1.b. This complementary structure will have interchanged Sparameters with the original one according to the Babinet's principle. As result, a reflector with similar behavior as the grounded FSS can be obtained. It should mention that the complementary unit cell is printed on foam substrate with thickness of $1.56 \mathrm{~mm}$.

\subsection{Antenna above the proposed reflectors}

For better evaluation of the proposed design concept, UWB planar antenna is used as radiator. The original design of this UWB planar antenna has been proposed in [3]. It is a CPW-fed circular disc antenna printed on dielectric substrate as shown in Fig. 1.c. In this paper, the employed substrate is RO4350B with dielectric constant of 3.48 and thickness of 1.524 and the dimensions of the CPW line are wf and sf where the former is the width of the line and the latter is the gap between the main line of the CPW and the ground plane and s is the slot gap between the circular patch and the ground plane. The antenna is located at distance $h$ above the grounded FSS reflector and the complementary FSS as indicated in Fig. 1.c. In each case, the reflection coefficient of the UWB antenna and its peak gain are computed and they will be discussed in the next section.

\section{Numerical results}

Many parameters affect the overall performance of the combined structures. Therefore, the effects of all those parameters have been studied thus their optimized values that give the best performance in terms of the matching band and the maximum gain, are selected, which are (in $\mathrm{mm}$ ): $\mathrm{W}=\mathrm{W} 2=5, \mathrm{~g}=0.25, \mathrm{La}=\mathrm{Wa}=$ $50, \mathrm{r}=14.5, \mathrm{wf}=3, \mathrm{sf}=0.28, \mathrm{~s}=0.35, \mathrm{~h}=15.5$, with overall reflectors size of $115.5 \mathrm{~mm}$ ( 22 cells). Fig. 2 illustrates the reflection coefficient of the UWB antenna amounted above the proposed reflectors and the peak gain obtained over frequency. Fig. 3 indicates the radiation patterns in the principal planes. These figures contain also the corresponding results of the UWB antenna without reflectors to allow a clear visualization of the achieved improvement owing to the reflectors. These results prove that the provident choice of the different parameters gives an antenna with operating band from 3.1 to $10.6 \mathrm{GHz}$ with quasi-constant maximum gain of average $9 \mathrm{~dB}$. It can also be noticed that the antenna shows a similar behavior at the distance $h$ from the both reflectors across the studied band except at $10 \mathrm{GHz}$ where a gain enhancement of $0.5 \mathrm{~dB}$ can be observed. However, the antenna still has a maximum gain variation of $0.5 \mathrm{~dB}$ across the entire UWB band in the two cases. The computed radiation patterns show the good effects of the reflectors on the radiation behavior of the antenna and confirm the gain enhancement (narrower main beams) which is meanly because of the minimization of the back radiation. Following the proper design methodology, similar results can be obtained using other UWB monopole antennas as radiators. 


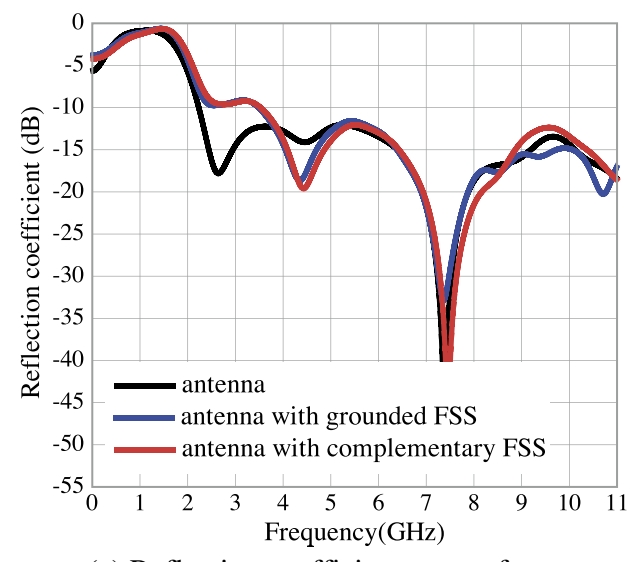

(a) Reflection coefficient versus frequency

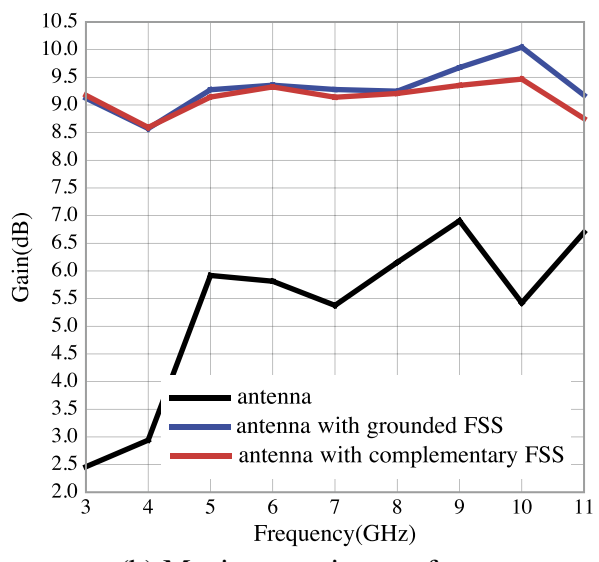

(b) Maximum gain over frequency

Fig. 2. Reflection coefficient and maximum gain of the UWB antenna with and without reflectors.

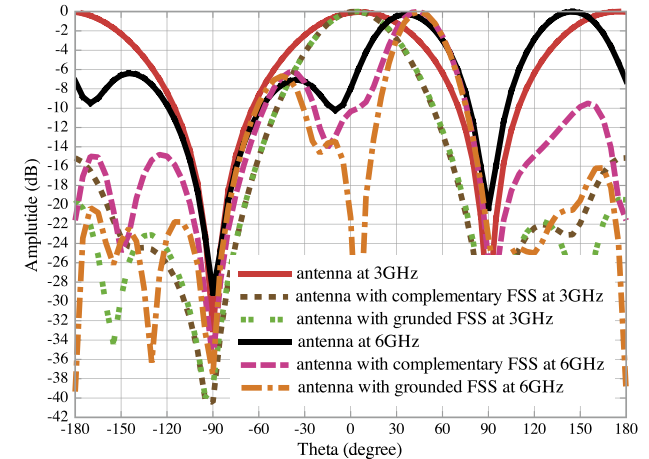

(a) Radiation patterns in E plane

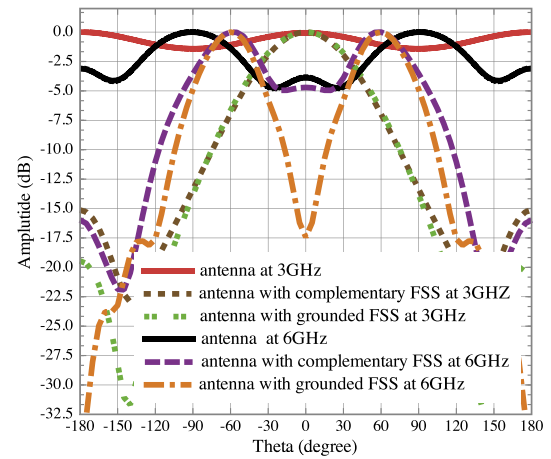

(b) Radiation patterns in $\mathrm{H}$ plane

Fig. 3. Radiation patterns at $3 \mathrm{GHz}$ and $6 \mathrm{GHz}$ for the antenna with and without the proposed reflectors.

\section{Conclusion}

In this letter, a technique to gradually increase the gain of UWB planar antennas over the frequency, by using FSS with low-profile sub-wavelength unit cells, has been proposed, which eliminates the restriction of the UWB planar antennas to be used only for one to multi-users applications and extends their potential applications to include the ones where stable and high gain is required. The effectiveness of the proposed FSS based reflectors is proved by means of using CPW-fed circular-disc monopole as a radiator. Maximum gain of $9 \mathrm{~dB}$ with maximum variation of $0.5 \mathrm{~dB}$ across the UWB band has been achieved while maintain the wide bandwidth of the used radiator. 